\title{
¿DE QUE MODO EL LIBRO DE HABACUC RESISTE A LA VIOLENCIA?
}

\author{
Una propuesta hermenéutica
}

Nos proponemos en el artículo estudiar la respuesta del libro de Habacuc al problema de la violencia suscitada en la historia como consecuencia del imperialismo y la prepotencia del poder. Realizamos una lectura de todo el libro, a partir de la estructura que consideramos para el mismo, observando los puntos álgidos que sugieren cualquier tipo de violencia. Este tema estudiado, nos surgió hace tiempo, como consecuencia del análisis textual de 1,12 , que implicaba posturas diferentes; por eso, damos cuenta de ese problema textual, de nuestra propuesta de resolución y de las consecuencias hermenéuticas significativas en el mensaje del libro. Al final del artículo, volvemos a centrarnos en el problema de la violencia, analizando la propuesta y el valor teológico del libro.

\section{Lectura y estructura del libro de Habacuc}

En este libro se plantea el problema de la justicia de Dios: una teodicea. El enfoque del planteo se lleva a cabo por medio de las quejas y las preguntas realizadas por el profeta al Señor, a propósito de la violencia y la injusticia suscitada en la historia. 
El libro es plausible pensarlo como una liturgia profética preparada para su celebración en el Segundo Templo. El Salmo final con sus anotaciones musicales, sería la prueba patente de este uso. ${ }^{1}$ Todo el libro se desarrolla en torno a la querella planteada por el profeta a propósito de la invasión Caldea. La estructura general del libro sería la siguiente:

- Título del libro $(1,1)$

- Primera lamentación por la sociedad judaíta (1,2-4)

- Primera respuesta del Señor (1,5-11)

- Segunda lamentación de Habacuc $(1,12-17)$

- Decisión de Habacuc de esperar vigilante una respuesta $(2,1)$

- Segunda respuesta del Señor (2,2-5)

- Ayes de condena a la criminal prepotencia de Babilonia, como interpretación profética de lo dicho por el Señor $(2,6-$ 20)

- Epílogo: un salmo como representación cúlticosapiencial de todo el libro (3,1-19)

Presentamos a continuación, una lectura del libro, que se detiene en las referencias a la violencia, y sobre todo, a como ésta se va incrementando y aumentando escandalosamente.

\section{- Título del libro $(1,1)$}

Todo el libro queda orientado por el sentido que le brinda la primera palabra de su título: maśśa $\bar{a}$ ', que la traducimos por "oráculo", en referencia al género profético (cf. Is 13,$1 ; 15,1 ; 17,1 ; 19,1 ; \mathrm{Na} 1,1 ; \mathrm{Za}$ $9,1 ; 12,1 ;$ Ma 1,1$)$. No hay que perder de maśśa $\bar{a}$ ' su sentido propio y principal: carga (Is 22,25), peso (Ex 23,5), trasporte agobiante (Is 46,1; $2 \mathrm{Cr} 35,3)$, que indicaría también la carga del mensaje que el libro contiene. El verbo $h \bar{a} z \bar{a} h$ - ver o contemplar, refuerza el mensaje que el libro contiene, fundamentado en la experiencia auricular y visual del profeta.

\section{- Primera lamentación por la sociedad judaita (1,2-4)}

En el primer lamento $(1,2-4)$, el profeta en dos oportunidades clama por la hâmās, primero en el v. 2; y luego en el v. 3 en dupla con šod. En su primera aparición hăamass se refiere a la violencia denotando un

1 M. FLoYd, Minor Prophets. II, Michigan, Eerdmans, 2000, pp. 641-642; J. WATTS, "Psalmody in Prophecy. Habakkuk 3 in Context», en J. WATTS / P. HOUSE 
sentido criminal que implica fuerza y atropello físico del inocente. En el v. 3 aparece en bina con otra palabra sinónima muy sugestiva: šod $w^{e}$ $h \bar{a} m \bar{a} s$ (destrucción y violencia) ${ }^{2}$ la dupla funciona siempre como una endiadis para mostrar la totalidad del mal cometido, (cf. Is 60,$18 ; \mathrm{Jr}, 6,7$; 20,8; Ez 45,9; Am 3,10).

En el v. 3, también aparece otra dupla de palabras 'āven y 'àmāl (iniquidad y miseria) $)^{3}$ se suele utilizar en contextos donde se reclama o pronuncia un juicio justo (cf. Nm 23,21; Is 10,1; 59,4; Sal 7,15; 10,7; $90,10 ; \mathrm{Jb} 4,8 ; 5,6 ; 15,35)$. Leyendo la bina en un sentido englobante, " 'àmāl contiene una cierta especificación que lleva a concretar la fuerza nefasta y negativa de 'āven en términos de un opresión injusta." ${ }^{4}$ En concreto, la realización de la iniquidad produce miseria, ese sería el sentido global dado por la dupla.

El v. 4 es introducido por la partícula conectora " "al'kēn" que funciona de forma consecutiva, por eso la cadena de violencia descripta antes "paralizas la ley". La referencia es al comportamiento de la sociedad judaíta pre exílica. Habacuc presenta su queja al Señor buscando respuestas que le expliquen "hasta cuando" el malvado asediará al justo, porque la mišpāt se pervierte por las maquinaciones y las violencias de los más poderosos que paralizan la ley.

\section{- Primera respuesta del Señor (1,5-11)}

El Señor responde al profeta con un primer oráculo (1,5-11) en el que promete movilizar al pueblo Caldeo, esta acción se motiva, supuestamente, en la erradicación de la maldad. El tema es que las consecuencias parecen ser peores. El pueblo Caldeo es descripto con el adjetivo "mar" y con el participio nifal del verbo "māhar", es decir, son

2 HALOT describe la bina como una expresión estereotipada y propone traducir como "muerte y destrucción" (cf. "II Tש").

3 ‘āmāl se puede traducir por «trabajo» pero tiene el sentido de fatigoso, de agobiante, de opresivo. Cf. Qo 1,3 (como sustantivo); 2,21.22; 4,6, etc. Cuando se utiliza en el lamento contra los enemigo 'āmāl indica con frecuencia la actuación

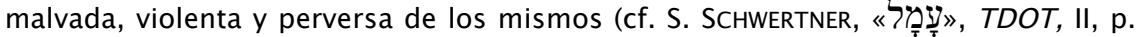
426).

4 P. JARAmillo Rivas, La injusticia y la opresión en el lenguaje figurado de los profetas. Estella, Verbo Divino, 1992, 276.

5 tāpûg lo traducimos como "paralizar", dado que el verbo pûg, por un lado implica entorpecimiento para el movimiento (cf. Sal 38,9); y por el otro, insensibilidad -en el sentido de enfriar las pasiones o no perturbarse- (cf. Gn 45,26). Con el verbo paralizar buscamos expresar los dos matices posibles, que por otro lado, bien se adaptan al contexto de la unidad literaria. 
"siniestros e impetuosos" (cf. v. 6) recorren la tierra para usurpar las casas ajenas. Lo más terrible se dice en el v. 7, ellos son temibles por que imponen su propia mišpāt. El oráculo continúa con una descripción bastante extensa de la ferocidad y la crueldad del ejército caldeo (cf. vv. 8-11), se vuelve a insistir en que su llegada genera violencia (cf. v. 9), y se concluye que "su fuerza" -el motor dinamizador de su violencia- es vista como su propio dios (cf. v. 11).

El oráculo no responde a los cuestionamientos de Habacuc, al contrario dará pie a una querella más álgida en la que se le cuestionará a Dios su justicia de modo más claro.

\section{- Segunda lamentación de Habacuc $(1,12-17)$}

En esta lamentación (1,12-17) encontramos el problema textual al que nos referimos al comienzo del artículo. Es interesante estudiarlo, debido a que la opción que se tome genera dos significados diferentes, que acarrean influencias hermenéuticas distintas para la comprensión de todo el libro; por lo menos, respecto al punto de vista que proponemos para su lectura. Desarrollamos, a continuación, este problema textual, porque la opción seguida, repercute en el planteo exegético que vamos a presentar al final del artículo.

En 1,12, en el TM leemos lo' nāmût "no moriremos". La tradición judía de lectura recoge en la corrección de los escribas, conocidos como los tiqqûnê sôferîm, lo’ tāmût "no mueres” ¿Cuál es la lectura original? La preservada en la lista de los tiqqûnê sôferîm, o la del TM. Para llegar a precisarlo es importante intentar explicar cuáles pueden haber sido las razones, por las cuáles una de las dos lecturas fue sentida como dificultosa.

Si partimos de la base que la LXX sigue la misma lectura que el TM parecería innecesaria la investigación, ${ }^{6}$ puesto que, si ésta versión tan importante presenta la misma lectura que el TM, como justificar la validez de la propuesta de los tiqqûnê sôferîm. Igualmente, la lectura de éstos tiene bastante peso, ya que si revisamos las traducciones más divulgadas en Latinoamérica, vemos que de siete versiones cinco de ellas siguen la propuesta de los antiguos escribas y solo dos la del TM:

6 En LXX leemos: mè apothánōmen. En 1QpHab no podemos observar el texto pues las primeras 9 palabras de este versículo se perdieron, presumiblemente, en la laguna de la parte inferior del otro manuscrito. Lo que se conserva en el pesher es el comentario del texto: "Dios no destruirá a su pueblo por mano de las naciones", que claramente presupone el TM. Se puede ver [en línea]: <http://www.moellerhaus.com/pesher5.htm> [consulta abril de 2012]. 
- ¿No eres tú, Señor, desde antiguo mi Dios santo que no muere? (Biblia del Peregrino o de Nuestro Pueblo, 1995)

- ¿No eres tú desde antiguo, Yahvé, mi Dios, mi santo? ¡Tú no mueres! (Biblia de Jerusalén, 2 da ed. 1975, 3ra ed. 1998 y 4ta ed. 2009)

- Mi Dios, mi Santo ¿no eres tú el Yavé de antes que no puede morir? (Biblia Latinoamericana, 1ra ed. 1972 y $120 v a$. ed. 2005)

- ¿No eres tú, Señor, desde los tiempos antiguos, mi Dios, mi Santo, que no muere jamás? (El Libro del Pueblo de Dios, 1ra ed. 1981 y 21 va ed. 1999)

- Señor, ¿acaso no existes tú eternamente, mi Dios santo e inmortal? (Dios Habla Hoy, 1994)

- ¿No eres tú desde el principio, Jehová, Dios mío, Santo mío? No moriremos. (Reina-Valera, 1995)

¿No eres tú, Señor, desde antiguo mi Dios, mi Santo? ¡No moriremos! (Biblia de América, 10ma ed. 1997)

Queda en evidencia la influencia que ha tenido la lectura de la tradición escribal para la mayoría de las traducciones presentadas, e incluso para el mismo K. Elliger, organizador del aparato crítico de la Biblia Hebraica Stuttgartensia que propone leer como los tiqqûnê sôferîm. La tradición judía recoge en total 18 casos de corrección de estos antiguos escribas. La razón fundamental que se argumenta para explicar los cambios de los tiqqûnê sôferîm tiene que ver con motivos teológicos. ${ }^{7}$ La piedad de los mismos, los llevaba a cambiar textos considerados irreverentes. En este caso de $\mathrm{Ha}$ 1,12, estarían armonizando el paralelismo, pues por un lado continúan hablando en el segundo estico de la misma persona (YHWH) y por otro, entendiendo el paralelismo de forma sinonímica (ser desde... no morir nunca) confesarían también la eternidad de Dios como uno de sus atributos principales:

"¿No eres tú desde antiguo YHWH?

mi Dios, mi Santo ¡Tú no mueres!”

Hoy día proponen varios que las correcciones pertenecen más a la tradición del midrash que a la de la masora, que son interpretaciones y no lecturas; por lo tanto, no se las toman como un signo de crítica textual.

Francis Andersen argumenta como posibilidad que los escribas contasen con otra versión del texto hebreo que contenía el testimonio de los tiqqûnê sôferîm. Su mirada jerarquiza a la variante textual del TM. Por otro lado, para reforzarlo, recuerda que las historias cananeas de Baal

7 Cf. C. MCCARTHY, The Tiqqune Sopherim and Other Theological Correction in the Masoretic Text of the Old Testament. Göttingen, Vandenhoeck \& Ruprecht, $1981,15.111$. 
cuentan como este dios muere y es restaurado a la vida. ${ }^{8} \mathrm{Su}$ propuesta para el texto de Habacuc es que estamos ante un epíteto ("YHWH es el Dios viviente") que debe haber significado vivir para siempre, justamente resaltando el sentido opuesto de nunca morir.

Si analizamos la oración del v. 12, el verbo sím, que sigue a lo' $n \bar{a} m \hat{u} t$, es un qal perfecto en 2 da persona masculina singular. Este verbo $s^{\prime} a m^{e}$ tô invita a adoptar la enmendación con lo' nāmût para que coincidan las conjugaciones. No parece suficiente el argumento para justificar el cambio, dado que no es raro el contraste de personas realizado mediante las conjugaciones verbales (cf. Sal 44,4-5; Mi 2,4), ${ }^{9}$ por eso, la diferencia de número, al no alterar la gramática, no es una causa sustentable -en este caso- para justificar el cambio.

Es conveniente observar las razones que pueden provenir de la estructura y del género literario del texto. Si la frase se encuentra en la segunda lamentación de Habacuc, en la cual el profeta responde al primer oráculo del Señor. No es lo mismo leer esta queja con cualquiera de las dos frases; pues en la estructura de 1,12-17 no expresan las dos lo mismo.

lo' nāmût es una oración negativa verbal simple ${ }^{10}$ que funciona en el contexto como afirmación de confianza. El elemento de confianza funciona en las lamentaciones como reproche retórico. Suele referirse a la experiencia del pasado salvífico que crecía en un grupo muy unido con Dios (cf. el funcionamiento de otros reproches retóricos similares: Sal $22,4-6 ; 31,16 ; 40,18 ; 56,4-5 ; 63,2 ; 89,27) .{ }^{11}$ La inclusión de una afirmación de confianza, junto a la queja de la lamentación (1,13-17) aumenta las expectativas del problema teológico que debe resolverse, “¿Por qué ves a los traidores y callas cuando el impío traga al que es más justo que él?", sobre todo sabiendo que "tus ojos puros no pueden ver el mal, que eres incapaz de contemplar la opresión" (cf. 1,13). La afirmación confiada, puesta junto al lamento, solo sirve para profundizar el drama.

${ }^{8}$ En el POA son típicos estos dioses. Cf. F. ANDERSEN, Habakkuk. New York, Doubleday, 2002, p. 177.

9 Cf. A. VAN DER WAL, «lo' nāmût in Habakkuk I 12: A Suggestion», en VT 38/4 (1988) p. 482.

10 La partícula negativa lo' se usa especialmente en las oraciones verbales delante de las formas finitas. La posible lectura de la oración en volitivo "que no muramos", que obviamente cambiaría el sentido de la perícopa, no es posible; pues con las formas volitivas yusivas y cohortativas se utiliza la partícula 'al. Cf. JoüON MURAOKA, §160 bf.

${ }^{11}$ Cf. E. Gerstenberger, Psalms I. Michigan, Eerdmans, 1991, p. 244. 
Si la declaración de confianza fuese "tú no mueres", el discurso sería mucho menos punzante y arriesgado. La frase tendría que haber sido reforzada con más sarcasmo pues así la razón parece objetable; ya que por el hecho que Dios no muera no se evita la muerte del pueblo. El escándalo de la lamentación no solo se provoca porque los ojos puros del Señor "no pueden ver el mal"; sino sobre todo, porque el Señor siempre cuidó de su pueblo, y los que viven la justicia no pueden morir (cf. 1,4). En el reproche y en la declaración de confianza se trasluce el conocimiento "desde antiguo" del Señor (miqqedem), un conocimiento lleno de experiencia y de intimidad con el profeta, quizás por eso decidirá plantantarse en la muralla esperando una respuesta.

Respecto al resto de la sección, encontramos varios elementos literarios propios de las lamentaciones:

- qedem (antiguo) confesado como atributo: del Señor (cf. Sal 74,12); de Israel el pueblo adquirido (cf. Sal 74,2); de las obras o gestas del Señor (cf. Sal 44,2; 77,12; 143,5; Is 51,9).

- Las preguntas con un estilo directo y desafiante donde se deja en evidencia el abandono de Dios. En este caso está introducida por lāmāh (v. 13 cf. Sal 22,$1 ; 74,11 ; 79,10)$.

- Se suelen distinguir tres dimensiones de quejas en la lamentación: el propio sufrimiento, las acciones enemigas y la negligencia de Dios. Las tres se encuentran en el texto: las acciones enemigas (cf. vv. 12c.15-17) y la negligencia de Dios (cf. vv. 12c.13-14) se desarrollan juntas y ocupan casi toda la extensión del lamento. Respecto al propio sufrimiento lo encontramos denotado junto al grupo representado en el justo que es comido por los impíos (v. 13b).

- Suelen encontrarse como ya dijimos a propósito del análisis textual, fórmulas que funcionan como afirmaciones de confianza, con un sentido exquisitamente retórico y en un estilo directo, ponen las aflicciones en las manos de Dios. En el caso de esta perícopa postulamos la frase que motivó nuestra exposición anterior lo' nāmût (v. 12b).

\section{- Decisión de Habacuc de esperar vigilante una respuesta $(2,1)$}

La lamentación finaliza con una declaración que hace el profeta $(2,1)$. En actitud de súplica, se dispone a escuchar afirmándose en su atalaya, aparentemente con una extensa vigilia, ${ }^{12}$ como un centinela que "mira atentamente" preparándose él mismo y a la vez al lector, para la escucha del Señor. La fórmula es similar a la del vigilante de Is que

12 Esa sensación dejan los tres yiqtol cohortativos con un matiz de fuerza en este caso utilizado para expresar el convencimiento de esperar la respuesta lo que sea necesario. 
manifiesta su expectativa ante la inminente caída de Babilonia (cf. Is 21,8-9).

\section{- Segunda respuesta del Señor (2,2-5)}

La lamentación recibirá, al igual que la anterior, un segundo oráculo como respuesta (2,2-5), que pone en contraste la fidelidad del justo con el codicioso (vv. 4-5).

Los vv. 4-5 deben comprenderse juntos pues están conectado sintácticamente con las partículas conjuntivas $w^{e}$ ap $k \hat{\imath}$ (ciertamente, además). La mayoría de los autores unen el v. 5 al v. 6 indicándolos como preludio de la sección de los "ay" y justificando que el oráculo terminaba originalmente en el v. $4 .^{13}$ Nos parece más apropiado comprender el v. 5 a la luz de lo anunciado por el v. 4 que anticipaba que "sucumbirá el que no tiene el alma recta". Y si bien es plausible que el v. 5 sea un agregado posterior, en esa intención redaccional de unir los vv. 4 y 5 , también hay un sentido teológico, que propuso algún editor, y que nos lleva a respetar así esa unidad literaria.

El v. 5 compara al hombre orgulloso con el vino, por lo traicionero que esconden (cf. Pr 20,1 comparación similar) y se describe insaciable su ambición "que ensancha sus fauces como el sheol para acaparar naciones y pueblos" sin saciarse nunca. Es ese hombre del v. 5 a quien se describe en el v. anterior como el "que no tiene el alma recta". La violenta perversidad que moviliza a los caldeos que recorren "las anchuras de la tierra para adueñarse países ajenos" $(1,6)$, parece tener origen en el orgullo del poder y en la codicia originada por las riquezas. Se promete para ellos el derrumbe, sucumbirán por su delito, solo vivirán los justos debido a su fidelidad a la Ley del Señor.

- Ayes de condena a la criminal prepotencia de Babilonia, como interpretación profética de lo dicho por el Señor (2,6-20)

La sección siguiente compuesta por cinco ayes ${ }^{14}$ "está colgada de la sección anterior, como copla satírica que entonarán todos los pueblos

13 Cf. por ejemplo: R. HAAK, Habakkuk. Leiden, Brill, 1992, p. 59; M. SWEENEY, «Structure, Genre and Intent in the Book of Habakkuk» en VT 41 (1991) p. 71; R. CoGGins - J. HAN, Six Minor Prophets. Nahum, Habakkuk, Zephaniah, Haggai, Zechariah and Malachi. Oxford, Wiley-Blackwell, 2011, p. 65.

14 Este tipo de oráculos son la variante principal y más frecuente de los oráculos de condenación. Para un conocimiento exhaustivo del género literario 
contra el ambicioso insaciable." 15 La sección funciona como invectiva contra los malvados, y a la vez es una defensa de los pobres (cf. 2,6-20). El conjunto está unificado, a pesar que hayan sido encadenados editorialmente, fueron armados en una composición simple y en su efecto conjunto son una adecuada respuesta a la oración del profeta. ${ }^{16}$ Cada aye anuncia el delito y su condena consecuente. Por lo tanto, conforman pequeñas escenas episódicas que funcionan como confirmación de lo anunciado en el v. 5, que el malvado sucumbirá.

En el cuarto ay! (2,15-17), aparece nuevamente la dupla hâmās / šod, señaladas aquí como la retribución por las violencias y asesinatos realizados por Babilonia contra el Líbano (metáfora isaiana de Israel cf. Is 2,$13 ; 14,8 ; 29,17 ; 33,8-9 ; 35,2)$. La barbarie cometida por Babilonia se le vuelve toda encima y será mucho más grande que su gloria obtenida $(2,16 b)$, como copa de ira que da de beber el Señor, típica metáfora de su juicio soberano (cf. Sal 75; Is 51,17).

El libro podría haber concluido en el capítulo 2, con el v. 20 final, que llama a toda la tierra a un sobrecogido silencio, porque YHWH anunció en la visión su venida, y ahora está sentado en su Templo santo, y desde ahí vendrá. Al no suceder así, este último versículo prepara al lector para la recitación del Salmo, dejándolo con la mirada dentro del Templo, taciturno, delante de YHWH.

\section{- Epílogo: un salmo como representación cúltico-sapiencial de todo el libro $(3,1-19)$}

Finalmente, el libro presenta el salmo $(3,1-19)$ que continuando a la sección anterior que finalizaba con el Señor en su Templo y toda la tierra en un sobrecogido silencio $(2,20)$, dispone, de ese modo, a los lectores a la celebración cultual de esta lucha entre el Señor y los codiciosos, pero representados por diversos símbolos de origen mítico que evocan textos acadios de las luchas de Baal para establecer el orden (cf. vv. 3-15). ${ }^{17}$ En este punto, algunos autores han discutido largamente, respecto al background de Ha 3. Entendido para algunos desde la

«ayes» cf. C. WeStermann, Basic Forms of Speech. Philadelphia, The Westminster Press, 1966, pp. 190-198.

15 J. ÁBREGO, «Habacuc: el profeta en su puesto de guardia», en RevBib 60 (1998) p. 113.

16 Cf. ANDERSEN. Habakkuk, p. 225.

17 Se puede ver el mito: "El palacio de Baal" y "La lucha entre Baal y Yammu". Cf. V. Matthews - D. Benjamín, Paralelos del AT. Santander, Sal Terrae, 2004, pp. 244ss. 
mitología babilónica ${ }^{18}$ y para otros desde la cananea. ${ }^{19}$ El Salmo utiliza varias imágenes míticas que lo describen a YHWH enfrentando con violencia a los enemigos: marcha con su ejército 3,5; cabalga los carros 3,8; dispara el arco 3,9a; parte la tierra 3,9b; lanza flechas y empuña la lanza 3,11 ; pisa las naciones 3,12 ; destroza al malvado 3,13 ; perfora las cabezas defendiendo al pobre 3,14; camina sobre las aguas 3,15. El Señor es presentado así, como un Guerrero, "la descripción de la marcha y ataque de los caldeos contra las naciones en 1,5-11 tiene su contraparte antitética en la teofanía de 3,3-15, describiendo la marcha del Señor desde el sudeste (3,3-7) y su ataque como Warrior divino contra los poderes del caos (3,8-15)." ${ }^{, 20}$ El salmo celebra el conflicto y victoria de YHWH sobre los poderes hostiles. Este enfrentamiento es representado con la batalla del Señor contra las aguas, utilizando los temas de la creación, el diluvio y el éxodo como plataforma poética para narrar con forma hímnica la victoria del Señor. A pesar que el enemigo no es reconocido con un nombre propio -y gracias a esto el texto puede ser releído y actualizado en cualquier circunstancia- todos los autores reconocen que en primera instancia se trata de los caldeos.

El salmo finaliza con un testimonio del profeta, en el que primero expone sus experiencias físicas provocadas por la audición del Señor. $\mathrm{La}$ segunda parte del testimonio es una confesión en la que se pinta una situación de absoluta desolación, expresada como una carestía en la tierra $(3,17)$. El testimonio concluye con una fórmula de confianza o voto $(3,18)$ y su consecuencia inmediata expresada con elementos motivantes de estilo hímnico (cf. Sal 18,34; 2S 22,34; Dt 33,29).

\section{La violencia y el mensaje de Habacuc}

A partir de lo expuesto, se puede observar como en el libro de Habacuc se plantea una cadena de violencia, que si no es interrumpida, puede continuar hasta el infinito. La trama planteada por el libro invoca

18 W. IRWIN, «The Mythological Background of Habacuc Chapter 3», en JNES 15 (1956), pp. 47-50; F. STEPHENS, «The Babylonian Dragon myth in Habakkuk 3», en JBL 43 (1924), pp. 290-293.

19 J. DAY, God's Conflict with the Dragon and the Sea, Cambridge, University Press, 1988, pp. 104-9; B. BARRICK, «The Meaning and Usage of RKB in Biblical Hebrew», en JBL 101 (1982), pp. 492-493.

20 G. PRINSLOO, «Yahweh the Warrior: An Intertextual Reading of Habakkuk 3» en OTE 14/3 (2001) p. 477. 
un abrupto final para la cadena de violencia y destrucción desarrollada. ${ }^{21}$ Al final de la cadena permanece YHWH como el último dispensador de violencia:

- En el capítulo 1, YHWH condena la violencia judahita y el atropello del más justo $(1,2-4)$ con la movilización de los caldeos $(1,5-11)$

- En el capítulo 2, YHWH condena la violencia caldea (2,6-20) por su desenfreno y prepotencia sin justicia $(1,15-17 ; 2,5)$

- En el capítulo 3, se celebra la violencia omnipotente de YHWH para salvar a los suyos $(3,12-15)$

La corrupción de Judá, trajo aparejado un costo elevadísimo, el levantamiento de Caldea, que desencadenó una feroz violencia ejecutada contra la tierra y sus habitantes (1,7-10). Se aplica igualmente a YHWH el capítulo 3, como a caldea el 1 y 2 . A pesar de que el salmo se exprese en un lenguaje mítico y que no nombre a los caldeos la arquitectura redaccional del libro y su trama demuestran que a ellos se refiere en un primer nivel de significación (3,16 habla de: $l^{e}$ am $y^{e}$ gîdenn $\hat{u}$ "el pueblo que nos ataca"). El Señor se levantó contra caldea para frenar el avance de su prepotencia. Mientras es común hablar de la violencia del Señor como justicia, una rígida observación del libro, revela que no puede ser distinguida una violencia justa de una violencia injusta y más todavía si el texto lo leemos desde una perspectiva cristiana coherente (cf. Mt 5,20-42, sobre todo v. 39a: mè antistếnai tố ponērō). ${ }^{22}$ El libro de Habacuc declara injusta la violencia de Judá y la de Caldea, pero justa la de YHWH.

La violencia de YHWH es la más severa -dado que es el último eslabón de la cadena-, la de Caldea es menos severa, y la de Judá es la más endeble. No nos referimos al declararla endeble a que sea inocente, sino a que es capaz de ser vulnerada por la que está por encima, en este

21 Nos inspiramos para el planteo de esta sección en el sugestivo estudio de: C. HEARD, «Hearing the Children's Cries: Commentary, Ethics, and the Book of Habakkuk», en Semeia 77 (1997) pp. 75-89.

22 "no resistan al mal", al respecto dice U. LUZ: "Mateo no pensó una aplicación primariamente política de la renuncia a la violencia. Pero tampoco se puede excluir la esfera política: el v. 41 alude a ella, como el v. 40 hace referencia a la esfera judicial. Lo cierto es que la renuncia a la violencia no es para la comunidad un asunto interno de un cenáculo, sino una exigencia y oferta hecha a todos los hombres. La no violencia y la renuncia al derecho definen, pues, la conducta de la comunidad ante el mundo, como comportamiento de unos discípulos que mueven a los hombres a alabar al Padre (Mt 5, 16)". El evangelio según san Mateo, Salamanca, Sígueme, 1993, p. 409. 
caso la caldea. Por lo tanto, la violencia más severa es juzgada inequívocamente justa.

\section{Respuesta tradicional}

La respuesta que da el libro de Habacuc al problema de la violencia generada por la codicia y el imperialismo, no encuentra el modo de despegarse de esta cadena de violencia. No hay en este libro una revelación novedosa del obrar de Dios en la historia contra la idolatría del poder. Parece que YHWH responde del mismo modo que los injustos judahítas y caldeos. Se continúa, en alguna medida, la teología del Éxodo en la que el Señor luchó contra el Faraón y se cubrió de gloria al vencerlo y dejarlo derrotado en el fondo del mar (cf. Ex 4,21-23; 5,1-2; 14,4.17$18 ; 15,1-5)$. El profeta Nahún incluso -el libro que antecede a Habacuc en el orden canónico del rollo de los Doce- contiene un mensaje similar; pues celebra la caída de Nínive, ciudad repleta de maldad (cf. Na 2,13; $3,1.4 .19$ ), que fue vencida por el Señor (cf. Na 2,14; 3,5-7), un Dios que no tolera la injusticia y cura a su pueblo (cf. Na 2,3). Será recién la apocalíptica, en el siglo II a.C., la que introduzca novedades en esta perspectiva, prometiendo una solución y una victoria para los justos y los débiles al final de los tiempos (cf. Dn 12,1-3); ya que anuncia que alguien venido de entre las nubes del cielo, parecido a un ser humano, al que se le dio poder eterno y un reino que no pasará (cf. Dn 7,13-14), establecerá este reino al que se someterán todos los soberanos de la tierra (cf. Dn 7,27).

Habacuc no resuelve el problema, las respuestas que ofrece son parecidas a las de la tradición. En realidad esa respuesta, probablemente, nunca existió. El libro de Habacuc se suma a la sinfonía de voces (Sal, Pr, Qo, Ez) y también al griterío ( $\mathrm{Jb}, \mathrm{Jr}, \mathrm{Lm}$ ) de tantos autores bíblicos que afrontaron el dilema del dogma de la retribución. El libro hace ingresar a sus lectores en un ámbito cultual, pues es una liturgia y está preparado para su celebración. El pueblo que lo celebra, confiesa que el Señor vuelve a salvarlos $(3,13)$, cada vez que Él es invocado por la fe y que Israel se congrega, haciendo memoria de sus obras $(3,2)$.

A pesar de que no hay en el libro de Habacuc una propuesta innovadora que corte con la violencia generada por el imperialismo, encontramos en este profeta el valor de su postura crítica. Su actitud de no callar aceptando sumisamente la respuesta tradicional. Habacuc no 
acata con docilidad lo que interpretó su tradición, al contrario, en el libro resalta sobre todo su desacato. Con sus preguntas y su vigilia en la muralla se subleva y se atreve así, a recorrer un itinerario personal de fe, a la espera de una respuesta que le devuelva la paz.

\section{4. “„No moriremos!”}

En el libro hay tres textos que ofrecen un sentido diferente al expuesto, separándose, de algún modo, de la violencia. Son éstos, en nuestra opinión, los puntos de significación más altos de la trama, en donde se revela su más noble significado: $1,12 \mathrm{a} ; 2,4 \mathrm{~b}$ y 3,19 .

El primero que presentamos es $2,4 \mathrm{~b}$. Se encuentra en el segundo oráculo y es introducido con un peculiar llamado a la esperanza: "aspira a la meta y no defrauda; si se atrasa, espérala, pues vendrá ciertamente, sin retraso" (2,3). Esa ansiada meta o fin - qēs, expresa el cumplimiento cierto de que Dios va a intervenir en la historia a favor de su pueblo. En ese plazo de espera, "sucumbirá el que no tenga el alma recta" $(2,4 \mathrm{a})$; por eso, solo queda para el justo, una sola posibilidad para sobrevivir a la tragedia, apegarse al Señor, unirse a Él, apoyarse en Él, creerle. Ese es el sentido de ’ěmûnātô en la sentencia final de 2,4b:

el justo por su fidelidad (integridad) vivirá.

'ěmûnātô lo traducimos como fidelidad o integridad, porque la ĕmûnāh, como atributo personal del hombre, es fidelidad a sus palabras y obras (cf. Jr 7,28; 9,2; Sal 37,3) y en su relación a Dios es firme adhesión a Él y a sus promesas. ${ }^{23}$ En el texto, esto implica que la vida se encontrará confiando en el Señor.

A parte de esta declaración sobre la necesidad de la fe para vivir y alcanzar la meta, en el libro aparecen otras dos declaraciones. La segunda en la que nos detenemos, es el v. final del libro. En 3,19 el salmo finaliza con una confidencia del profeta, de matiz hímnico:

YHWH, mi Señor, mi fuerza,

23 Cf. C. KeIL - F. DelitzSCH, Biblical Commentary on the Old Testament. Edinburgh, 1866, [en línea]: <http://kad.biblecommenter.com/habakkuk/2.htm> [consulta abril de 2012]. 
me puso los pies como las gacelas, para sobre mis alturas hacerme caminar.

El profeta reconoce a YHWH como su Señor y su fuerza. Esta confesión da lugar en el colon que sigue a la intervención del Señor en su vida realizando algo inédito, pone sus pies como el de las gacelas. El símil se explica en el colon final, es la posibilidad de "caminar por mis alturas", como las gacelas, que andan con mucha facilidad por las cumbres de las montañas. El profeta, en este caso también poeta, se refiere a la elevación que se realiza en su vida a partir de su relación con el Señor confesada en el v. anterior con un breve cántico de acción de gracias: "yo me voy a regocijar en $\mathrm{YHWH}$, me voy a alegrar en Dios, mi salvación" $(3,18) .^{24}$ La fe lo hace transitar por un camino nuevo que está por encima de la historia y de su devenir (cf. Dt 32,12-13). Las alturas le pertenecen al Altísimo (cf. Am 4,13; Mi 1,3), el poeta o profeta anda más cerca de Dios, pues le fue revelado su obrar. Esta stanza concluye el itinerario planteado en todo el salmo y, sobre todo, en todo el libro.

Finalmente, llegamos después de todo este itinerario a la frase que motivo nuestro estudio, el tercer texto que interpretamos con una plusvalía de significado es el primero en aparecer en la trama del libros. En 1,12 al comenzar Habacuc la segunda lamentación, con la que responde al primer oráculo divino, después de la pregunta retórica del comienzo en la que se indaga a sí mismo apelando a la identidad de su Dios, se responde a sí mismo a la pregunta con un grito comunitario:

“¿No eres Tú desde antiguo YHWH, mi Dios, mi Santo? ¡No moriremos!”

En medio del ascenso de los caldeos, que van destruyendo todo lo que encuentran a su paso $(1,9)$ el profeta deja escuchar la voz de un grupo de víctimas entre las que se encuentra implicado. Si nos preguntamos por la identidad de estas víctimas, se puede deducir, que se trata de los justos $(1,4)$, dado que los impios merecen y causan el levantamiento de los caldeos.

“No moriremos!" es una confirmación creyente en la cual la comunidad expresa su fe en el Dios que conoce desde antaño. "¡No moriremos!" porque el Señor no abandonará al pueblo que se adquirió

${ }^{24}$ Cf. H. GunKel, Introducción a los Salmos. Valencia, Edicep, 1983, p. 93. 
como herencia. Este grito testimonia la confianza de la comunidad, que a pesar de la oscuridad en la que está sumergida, puesto que no vislumbran razones, ni salida para la desgracia (cf. 3,17), confían con alegría en el Señor salvador (cf. 3,18).

\section{Conclusión}

Cualquiera puede dar respuestas, pero para hacer preguntas, se necesita de una genialidad única. El libro Habacuc tiene preguntas perennes, que todavía no hallaron una respuesta exacta. Muchos hombres de todos los tiempos se las siguen preguntando (cf. Ap 6,10). A pesar de no haber hallado una respuesta precisa, el libro responde con la fe, una fe que tendrá que seguir progresando hasta encontrar más plenitud. A pesar de eso, no corresponde descalificar a Habacuc "como un profeta teológicamente menor, sino que hay que interpretarlo y valorarlo en su trasfondo histórico implícito: En una situación de una identidad social completamente destruida a raíz de la aguda amenaza enemiga, crea Habacuc un texto erudito e impresionante, como también poéticamente creativo, para despertar la esperanza."25

Varios siglos más tarde, el apóstol Pablo citará este libro. Cuando presenta a una comunidad de cristianos residentes en Roma el Evangelio de Jesús - como fuerza de Dios para la salvación de los hombres- y argumenta, sobre la necesidad de creer en este Evangelio donde se revela la justicia de Dios. En su carta, Pablo citará como fórmula para su tesis teológica, una Autoridad tomada de la Escritura: "El justo por la fe (ek písteōs) vivirá" (cf. Rm 1,15-17). Algunas cosas se cambian de la cita, la ěmûnāh a Dios y a sus mandatos de la cual hablaba el profeta, se cambió por pistis; la comprensión de la justicia de Dios, ya no se entendió como violencia, sino como perdón gratuito para todos obrado por Jesucristo (cf. Rm 3,23-26), y finalmente, la muerte, que se experimentaba como irremediable derrota, ahora había sido devorada por la victoria perdiendo ya su aguijón (cf. Rm 8,31-39).

LEANDRO ARIEL VERDINI

25 D. MARKL, «Hab 3 in intertextueller und kontextueller Sicht», en Bib 85/1 (2004) p. 107. 
¿DE QUE MODO EL LIBRO DE HABACUC RESISTE A LA VIOLENCIA?

Buenos Aires

leandro_verdini@uca.edu.ar 